\title{
Research Article \\ On Sumudu Transform and System of Differential Equations
}

\author{
Adem Kiliçman, ${ }^{1}$ Hassan Eltayeb, ${ }^{2}$ and Ravi P. Agarwal ${ }^{3}$ \\ ${ }^{1}$ Department of Mathematics, Institute for Mathematical Research, Universiti Putra Malaysia, \\ UPM, Serdang, Selangor, 43400, Malaysia \\ ${ }^{2}$ Mathematics Department, College of Science, King Saud University, P. O. Box 2455, \\ Riyadh 11451, Saudi Arabia \\ ${ }^{3}$ Department of Mathematical Sciences, Florida Institute of Technology, Melbourne, FL 32901, USA \\ Correspondence should be addressed to Adem Kiliçman, akilicman@putra.upm.edu.my
}

Received 6 January 2010; Accepted 19 March 2010

Academic Editor: Ferhan Atici

Copyright (C) 2010 Adem Kiliçman et al. This is an open access article distributed under the Creative Commons Attribution License, which permits unrestricted use, distribution, and reproduction in any medium, provided the original work is properly cited.

The regular system of differential equations with convolution terms solved by Sumudu transform.

\section{Introduction}

A differential equation by itself is inherently underconstrained in the absence of initial values as well as boundary conditions. It is also well known that a differential equation along with the initial values or boundary conditions can be represented by an integral equation by using this integral representation, it becomes possible to solve the problem. However one of the most important achievements, and applications of integral transform methods is solving the partial differential equations (PDEs) of second order. For this purpose recently a new integral transform, which is called Sumudu transform, was introduced by Watugala $[1,2]$ and used by Weerakoon [3] for partial derivatives of Sumudu transform, provided the complex inversion formula in order to solve the differential equations in different applications of system engineering, control theory and applied physics. The convolution theorem of Sumudu transform was proved by Asiru in [4]. This new transform was applied to the solution of ordinary differential equations and control engineering problems; see [1, 5]. In [6], some fundamental properties of the Sumudu transform were established. In [7], this new transform was applied to the one-dimensional neutron transport equation. In fact the relationship between double Sumudu and double Laplace transforms were studied in $[8,9]$. Furthermore in [10], the Sumudu transform was extended to the distributions and some of their properties 
were also studied. Thus, there have been several works on Sumudu transform and applied to different kind of problems.

In this paper, we prove Sumudu transform of convolution for the matrices and use to solve the regular system of differential equations.

Throughout the paper we use a square matrix, $P=\left[P_{i j}\right]$ of regular system having size $n \times n$ of polynomials and the associated determinant, $\operatorname{det}[P]$. If $\operatorname{det}(P)$ is not the zero polynomial (which we write as $\operatorname{det}(P) \neq 0$ ), we have $\operatorname{deg}[\operatorname{det}(P)] \leq|N(P)|$ where $N(P)$ is the degree of the polynomials in the regular matrix $P$. The case of equality is so important that we make the following statement. We say that $P$ is regular if $\operatorname{det}(P) \neq 0$ and the condition

$$
\operatorname{deg}[\operatorname{det}(P)]=|N(P)|,
$$

where $N_{j}(P)$ considered as the highest power of the variable term that occurs in the $j$ th column of matrix $P$, that is,

$$
N_{j}(P)=\max _{\substack{1 \leq i \leq m \\ P_{i j} \neq 0}}\left[\operatorname{deg}\left(\left[P_{i j}\right]\right)\right]
$$

Next we extend the result given in [4] as follows. For each $i$ and $j$ we define $\Psi_{P}^{(i, j)}(x)$ to be the $1 \times N_{j}$ matrix of polynomials given by the matrix product

$$
\Psi_{P}^{(i, j)}(x)=\left(\frac{1}{x} \frac{1}{x^{2}} \frac{1}{x^{3}} \cdots \frac{1}{x^{N-1}}\right)\left(\begin{array}{cccccc}
a_{1} & a_{2} & \cdot & \cdot & \cdot & a_{N_{j}} \\
a_{2} & a_{3} & \cdot & . & a_{N_{j}} & 0 \\
a_{3} & \cdot & . & a_{N_{j}} & 0 & 0 \\
\cdot & \cdot & \cdot & \cdot & \cdot & \cdot \\
a_{N_{j}} & 0 & . & . & . & 0
\end{array}\right)
$$

where the $a_{k}$ are the coefficients of $P_{i j}$ and $P_{i j}=\sum_{k=0}^{N_{j}} a_{k} / x^{k}$. In terms of (7.1) in [4] we have

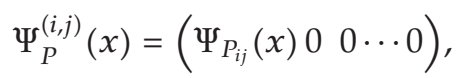

where the number of zero indicated is $N_{j}-\operatorname{deg}\left(P_{i j}\right)$. We define $\Psi_{P}$ to be matrix of polynomials and having size $m \times|N|$ defined in terms of the array of matrices:

$$
\Psi_{P}=\left(\begin{array}{cccc}
\Psi_{P}^{(1,1)} & \Psi_{P}^{(1,2)} & \ldots & \Psi_{P}^{(1, n)} \\
\Psi_{P}^{(2,1)} & \Psi_{P}^{(2,2)} & \ldots & \Psi_{P}^{(2, n)} \\
\Psi_{P}^{(3,1)} & . & \ldots & \Psi_{P}^{(3, n)} \\
\cdot & . & \ldots & \cdot \\
\Psi_{P}^{(m, 1)} & . & \ldots & \Psi_{P}^{(m, n)}
\end{array}\right)
$$


For each complex number $x, \Psi_{P}(x)$ define a linear mapping of $\mathrm{C}^{|N|}$ into $\mathrm{C}^{m}$. If any $N_{j}$ is zero, $\Psi_{P}^{(i, j)}$ is the empty matrix for all $i$ and the corresponding column of matrices in $\Psi_{P}$ is absent. If $N_{j}=0$ for all $j, \Psi_{P}(x)$ is defined to be the unique linear mapping of $\{0\}=\mathbf{C}^{0}$ into $\mathbf{C}^{m}$; its matrix representation is then the empty matrix. In particular consider

$$
\Psi_{P}(x)=\left(\begin{array}{cc}
x^{4}+2 x^{2} & 1-2 x \\
3 x^{4}+2 x & 4 x^{3}
\end{array}\right)
$$

Then we have $N_{1}=4, N_{2}=3$, and $N=(4,3)$. Thus $\Psi_{P}$ is the $3 \times 7$ matrix computed as

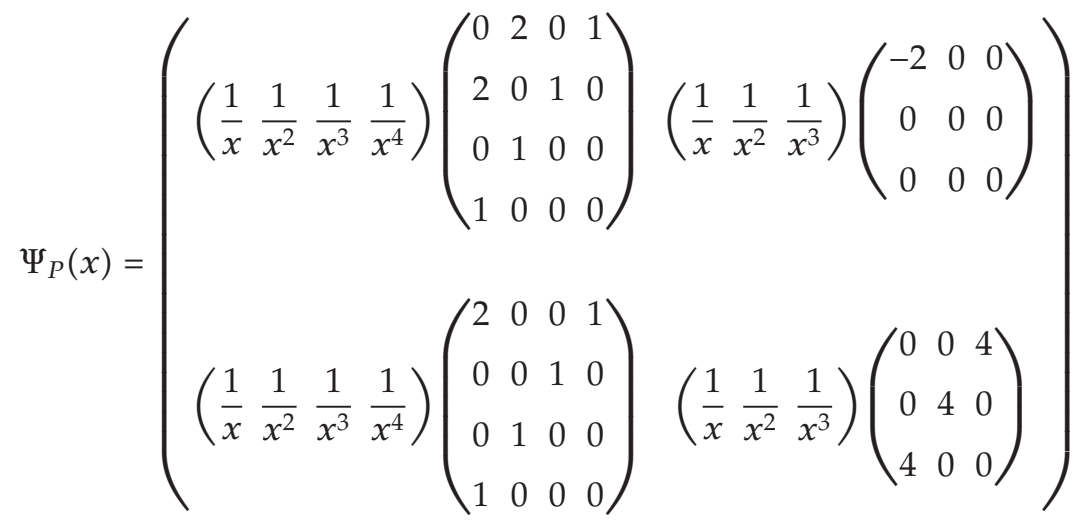

$$
\begin{aligned}
& =\left(\begin{array}{lllllll}
\frac{2}{x^{2}}+\frac{1}{x^{4}} & \frac{2}{x}+\frac{1}{x^{3}} & \frac{1}{x^{2}} & \frac{1}{x} & -\frac{2}{x} & 0 & 0 \\
\frac{2}{x}+\frac{1}{x^{4}} & \frac{1}{x^{3}} & \frac{1}{x^{2}} & \frac{1}{x} & \frac{4}{x^{3}} & \frac{4}{x^{2}} & \frac{4}{x}
\end{array}\right) .
\end{aligned}
$$

In general, if $f=\left(f_{1}, f_{2}, \ldots, f_{p}\right)$ is a sequence of functions on $(a, b), h=h_{1}, h_{2}, \ldots, h_{p}$ with each $h_{i}$ being an integer $\geq 0$ and $f_{i}$ being $h_{i}-1$ times differentiable on $(a, b)$, we will write, using the notation in [4],

$$
\begin{aligned}
& \Phi(f, a ; h)=\left(\Phi\left(f_{1}, a ; h_{1}\right), \Phi\left(f_{1}, a ; h_{2}\right), \ldots, \Phi\left(f_{p}, a ; h_{p}\right)\right) \in \mathbf{C}^{|h|} \\
& \digamma(f, b ; h)=\left(\digamma\left(f_{1}, b ; h_{1}\right), \digamma\left(f_{1}, b ; h_{2}\right), \ldots, \digamma\left(f_{p}, b ; h_{p}\right)\right) \in \mathbf{C}^{|h|},
\end{aligned}
$$

whenever the limits exist. If any $h_{i}$ is zero, the corresponding string is absent. If $h_{i}=0$ for all $i$ we define $\Phi(f, a ; h)=\digamma(f, b ; h)=0 \in \mathbf{C}^{0}$. The following proposition was proved in [4].

Proposition 1.1 (Sumudu transform of higher derivatives). Let $f$ be $n$ times differentiable on $(0, \infty)$ and let $f(t)=0$ for $t<0$. Suppose that $f^{(n)} \in L_{\mathrm{loc}}$. Then $f^{(k)} \in L_{\mathrm{loc}}$ for $0 \leq k \leq n-$ $1, \operatorname{dom}(S f) \subset \operatorname{dom}\left(S f^{(n)}\right)$ and, for any polynomial $P$ of degree $n$,

$$
S(P(\dot{D})(f))=P(u) S(f)(u)-M_{P}(u) \phi(f, n)
$$


for $u \in \operatorname{dom}(S f)$. In particular

$$
\left(S f^{(n)}\right)(u)=\frac{1}{u^{n}}(S f)(u)-\left(\frac{1}{u^{n}}, \frac{1}{u^{n-1}}, \ldots, \frac{1}{u}\right) \phi(f ; n)
$$

(with $\phi(f ; n)$ here written as a column vector). For $n=2$ we have

$$
\left(S f^{\prime \prime}\right)(u)=\frac{1}{u^{2}}(S f)(u)-\frac{1}{u^{2}} f(0+)-\frac{1}{u} f^{\prime}(0+)
$$

Now we want to extend the above proposition to the system of differential equations as follows.

Proposition 1.2. Let $(f)=\left(f_{1}, f_{2}, f_{3}, \ldots, f_{n}\right)$ be functions and let $(h)=\left(h_{1}, h_{2}, \ldots, h_{n}\right)$ be a sequence of integers $\geq 0$ such that $m \times n$, a matrix of polynomial $P$, satisfies $N(P)=h$. Furthermore for each $j$ we let $f_{j}$ be $h_{j}$ times differentiable on $(0, \infty)$ and we let $f(t)=0$ for $t<0$. Suppose that $f_{j}^{h_{j}} \in L_{\mathrm{loc}}$ for each $j$. Then $f_{j}^{r} \in L_{\mathrm{loc}}$ for $0 \leq r \leq h_{j}-1, \operatorname{dom}(S f) \subset \bigcap_{j=1}^{n} \operatorname{dom}\left(S f_{j}^{h_{j}}\right)$ and, we have,

$$
S(P(\dot{D})(f))(u)=P(u) S(f)(u)-\Psi_{P}(u) \phi(f, h) .
$$

In the following theorem we discuss the Sumudu transform of convolution of matrices.

Theorem 1.3. Let $A(t)=\left[f_{i j}(t)\right] \in M_{n}^{I}$ and $B(t)=\left[g_{i j}(t)\right] \in M_{n}^{I}$ be Sumudu Transformable. Then

$$
S[A(t) \circledast B(t)](u)=u S[A(t)] S[B(t)]
$$

where $M_{n}^{I}$ is the set of $n \times n$ matrices for whose entries are integrable.

Proof. Suppose that $S\left[f_{i j}(t)\right]=F_{i j}(u)$ and $S\left[g_{i j}(t)\right]=G_{i j}(u)$, by induction.

In case $n=2$, we have the following matrices:

$$
\begin{gathered}
A(t)=[f(t)]_{2 \times 2}=\left[\begin{array}{ll}
f_{11}(t) & f_{12}(t) \\
f_{21}(t) & f_{22}(t)
\end{array}\right], \\
B(t)=[g(t)]_{2 \times 2}=\left[\begin{array}{ll}
g_{11}(t) & g_{12}(t) \\
g_{21}(t) & g_{22}(t)
\end{array}\right] \in M_{2}^{I}
\end{gathered}
$$

as Sumudu transformable, then Sumudu transform of the above matrices are given by

$$
S\left[[f(t)]_{2 \times 2}\right](u)=\left[\begin{array}{ll}
F_{11}(u) & F_{12}(u) \\
F_{21}(u) & F_{22}(u)
\end{array}\right], \quad S\left[[g(t)]_{2 \times 2}\right](u)=\left[\begin{array}{ll}
G_{11}(u) & G_{12}(u) \\
G_{21}(u) & G_{22}(u)
\end{array}\right] .
$$


We have

$$
u S\left[[f(t)]_{2 \times 2}\right](u) S\left[[g(t)]_{2 \times 2}\right](u)=\left[\begin{array}{cc}
\alpha & \beta \\
\zeta & \eta
\end{array}\right]=S\left[[f(t)]_{2 \times 2} \circledast[g(t)]_{2 \times 2}\right](u),
$$

where

$$
\begin{array}{ll}
\alpha=u F_{11}(u) G_{11}(u)+u F_{12}(u) G_{21}(u), & \beta=u F_{11}(u) G_{12}(u)+u F_{12}(u) G_{22}(u), \\
\zeta=u F_{21}(u) G_{11}(u)+u F_{22}(u) G_{21}(u), & \eta=u F_{21}(u) G_{12}(u)+u F_{22}(u) G_{22}(u) .
\end{array}
$$

Similarly, in case $n=3$ it is also true that

$$
u S\left[[f(t)]_{3 \times 3}\right](u) S\left[[g(t)]_{3 \times 3}\right](u)=S\left[[f(t)]_{3 \times 3} \circledast[g(t)]_{3 \times 3}\right](u) .
$$

Assuming that $n=k$ is true for the case $n=k+1$, we have the following matrices:

$$
\begin{aligned}
A(t)=[f(t)]_{k+1 \times k+1} & =\left[\begin{array}{ccc}
f_{11}(t) & \cdots & f_{1 k+1}(t) \\
f_{21}(t) & \cdots & f_{2 k+1}(t) \\
\vdots & \cdots & \vdots \\
\vdots & \cdots & \vdots \\
f_{k+11}(t) & \vdots & f_{k+1 k+1}(t)
\end{array}\right] \in M_{k+1}^{I} \\
B(t)=[g(t)]_{k+1 \times k+1} & =\left[\begin{array}{ccc}
g_{11}(t) & \cdots & g_{1 k+1}(t) \\
g_{21}(t) & \cdots & g_{2 k+1}(t) \\
\vdots & \cdots & \vdots \\
\vdots & \cdots & \vdots \\
g_{k+11}(t) & \cdots & g_{k+1 k+1}(t)
\end{array}\right] \in M_{k+1}^{I}
\end{aligned}
$$

having Sumudu transforms given by

$$
S[A(t)]=S\left[[f(t)]_{k+1 \times k+1}\right](u)=\left[\begin{array}{ccc}
F_{11}(u) & \cdots & F_{1 k+1}(u) \\
F_{21}(u) & \cdots & F_{2 k+1}(u) \\
\vdots & \cdots & \vdots \\
\vdots & \cdots & \vdots \\
F_{k+11}(u) & \cdots & F_{k+1 k+1}(u)
\end{array}\right] .
$$


Similarly if

$$
S[B(t)]=S\left[[g(t)]_{k+1 \times k+1}\right](u)=\left[\begin{array}{ccc}
G_{11}(u) & \cdots & G_{1 k+1}(u) \\
G_{21}(u) & \cdots & G_{2 k+1}(u) \\
\vdots & \cdots & \vdots \\
\vdots & \cdots & \vdots \\
G_{k+11}(u) & \cdots & G_{k+1 k+1}(u)
\end{array}\right],
$$

then we have

$$
u S\left[[f(t)]_{k+1 \times k+1}\right] S\left[[g(t)]_{k+1 \times k+1}\right](u)=\left[\begin{array}{ccc}
\Phi & \cdots & \Gamma \\
\Theta & \cdots & \Lambda \\
\vdots & \cdots & \vdots \\
\vdots & \cdots & \vdots \\
\Upsilon & \cdots & \Omega
\end{array}\right],
$$

where

$$
\begin{aligned}
& \Phi=u F_{11}(u) G_{11}(u)+\cdots+u F_{1 k+1}(u) G_{k+11}(u) \\
& \Gamma=u F_{11}(u) G_{1 k+1}(u)+\cdots+u F_{1 k+1}(u) G_{k+1 k+1}(u) \\
& \Theta=u F_{21}(u) G_{11}(u)+\cdots+u F_{2 k+1}(u) G_{1 k+1}(u), \\
& \Lambda=u F_{21}(u) G_{1 k+1}(u)+\cdots+u F_{2 k+1}(u) G_{k+1 k+1}(u) \\
& \vdots \quad \ldots \quad \vdots \\
& \Upsilon=u F_{k+11}(u) G_{11}(u)+\cdots+u F_{k+1 k+1}(u) G_{k+11}(u), \\
& \Omega=u F_{k+11}(u) G_{1 k+1}(u)+\cdots+u F_{k+1 k+1}(u) G_{k+1 k+1}(u) .
\end{aligned}
$$

Thus,

$$
u S\left[[f(t)]_{k+1 \times k+1}\right](u) S\left[[g(t)]_{k+1 \times k+1}\right](u)=S\left[[f(t)]_{k+1 \times k+1} \circledast[g(t)]_{k+1 \times k+1}\right](u) .
$$

The proof completes. 
The inverse $P^{-1}(u)$ of $P(u)$ will exist provided that $u$ is not a root of the equation $\operatorname{det}[P(u)]=0$; hence let $\widetilde{P}$ denote the adjugate matrix of $P$; by elementary matrix theory we have

$$
[P(u)]^{-1}=\frac{1}{\operatorname{det}[P(u)]} \tilde{P}(u) .
$$

Proposition 1.4 (Solution of homogeneous equation of regular system). Let $P$ be regular and let $y$ be $N(P)$ times differentiable on $(0, \infty)$ and zero on $(-\infty, 0)$ and suppose that

$$
P(D) y=0
$$

then $y$ is given (except at 0) by the formula

$$
y=S^{-1}\left[[P(u)]^{-1} \Psi_{P}(u) \phi(y, N(P))\right]
$$

Proof. By using (1.12) and assuming that $y$ is Sumudu transformable, we have

$$
S(P(\dot{D})(y))(u)=P(u) S(y)(u)-\Psi_{P}(u) \phi(y, h)
$$

Equation (1.26) becomes

$$
P(u) S(y)(u)=\Psi_{P}(u) \phi(y, h),
$$

using (1.25)

$$
S(y)(u)=[P(u)]^{-1} \Psi_{P}(u) \phi(y, h)
$$

Finally by taking the inverse Sumudu transform of the above equation we have

$$
y=S^{-1}\left[[P(u)]^{-1} \Psi_{P}(u) \phi(y, h)\right],
$$

where we assume that the inverse transform exists.

The next proposition was proved in [4] for the single differential equation, and we extend it to the regular system of differential equations.

Proposition 1.5. Let $P$ be regular and let $b$ be the greatest of the real parts of the roots of the equation

$$
\operatorname{det}\left[P\left(\frac{1}{x}\right)\right]=0
$$


if $\operatorname{deg}[\operatorname{det}(P)]>0$ (otherwise put $b \rightarrow-\infty$ ). Let $f=\left[f_{i}\right]$ be continuous on $(0, \infty)$, and zero on $(-\infty, 0)$, locally integrable, and Sumudu transformable and furthermore suppose that

$$
P(D) y=f
$$

then we have

$$
P(u) S[(y)](u)=S(f)(u)+\Psi_{P}(u) \phi(f, N(P))
$$

for $1 / u>b$ in $\operatorname{dom}(S f)$.

Note that the most general system of equations can be written in the matrix form as

$$
\left[\begin{array}{cccc}
P_{11}(D) & P_{12}(D) & \cdots & P_{1 n}(D) \\
P_{21}(D) & P_{22}(D) & \cdots & P_{2 n}(D) \\
\vdots & \vdots & \cdots & \vdots \\
\vdots & \vdots & \cdots & \vdots \\
P_{n 1}(D) & P_{n 2}(D) & \cdots & P_{n n}(D)
\end{array}\right]\left[\begin{array}{c}
y_{1} \\
y_{2} \\
\vdots \\
\vdots \\
y_{n}
\end{array}\right]=\left[\begin{array}{cccc}
f_{11}(t) & f_{12}(t) & \cdots & f_{1 n}(t) \\
f_{21}(t) & f_{22}(t) & \cdots & f_{2 n}(t) \\
\vdots & \vdots & \cdots & \vdots \\
\vdots & \vdots & \cdots & \vdots \\
f_{n 1}(t) & f_{n 2}(t) & \cdots & f_{n n}(t)
\end{array}\right] *\left[\begin{array}{c}
g_{1} \\
g_{2} \\
\cdots \\
\cdots \\
g_{n}
\end{array}\right]
$$

which denotes the system

$$
\sum_{j=1}^{n} P_{i j}(D) y_{i}=\sum_{j=1}^{n} f_{i j} * g_{i} \quad(1 \leq i \leq n)
$$

Here the $P_{i j}$ are polynomials and if we let $P=\left[P_{i j}\right], f=\left[f_{i j}\right]$, respectively, and the vectors $y=\left[y_{i}\right], g=\left[g_{i}\right]$ with $n$ components, then the above equation can be written in the form of

$$
P(D) y=f * g
$$

under the initial condition

$$
y(0)=y_{0}, y^{\prime}(0)=y_{1}, \ldots, y^{(n-1)}(0)=y_{n-1}
$$

Since $y^{(k)}$ is locally integrable, thus, Sumudu transformable for $0 \leq k \leq n$ and for every such $k$, then Sumudu transform of (1.37) is given by

$$
P(u) S(y)(u)=u S(f)(u) S(g)(u)+\Psi_{P}(u) \phi(y, N(P))
$$


where $P(u)$ is a matrix defined by

$$
P(u)=\left[\begin{array}{cccc}
P_{11}\left(\frac{1}{u}\right) & P_{12}\left(\frac{1}{u}\right) & \cdots & P_{1 n}\left(\frac{1}{u}\right) \\
P_{21}\left(\frac{1}{u}\right) & P_{22}\left(\frac{1}{u}\right) & \cdots & P_{2 n}\left(\frac{1}{u}\right) \\
\vdots & \vdots & \cdots & \vdots \\
\vdots & \vdots & \cdots & \vdots \\
\vdots & \vdots & \cdots & \vdots \\
P_{n 1}\left(\frac{1}{u}\right) & P_{n 2}\left(\frac{1}{u}\right) & \cdots & P_{n n}\left(\frac{1}{u}\right)
\end{array}\right]
$$

and $\Psi_{P}(u)$ by (1.41).

In order to find the solution of (1.37), first of all we multiply (1.39) by the inverse matrix $P^{-1}(u)$, then we get

$$
S(y)(u)=[P(u)]^{-1}\left[u S(f)(u) S(g)(u)+\Psi_{P}(u) \phi(y, N(P))\right]
$$

Now, by taking the inverse Sumudu transform for both sides of (1.41)

$$
y(t)=S^{-1}\left[P(u)^{-1}[u S(f)(u) S(g)(u)]\right]+S^{-1}\left[P^{-1}(u) \Psi_{P}(u) \phi(y, N(P))\right]
$$

provided that the inverse exists for each term in the right-hand side of (1.42).

To illustrate our method, we give the following example.

Example 1.6. Solve for $t>0$ the system of two equations

$$
\begin{aligned}
& x^{\prime \prime}+2 y^{\prime}-2 x=-\sin (t), \quad x(0)=1, \quad x^{\prime}(0)=2, \\
& y^{\prime \prime}-2 x^{\prime}-2 y=\cos (t)-2, \quad y(0)=0, \quad y^{\prime}(0)=1 \text {. }
\end{aligned}
$$

The matrix

$$
P(u)=\left[\begin{array}{cc}
\frac{1}{u^{2}}-2 & \frac{2}{u} \\
-\frac{2}{u} & \frac{1}{u^{2}}-2
\end{array}\right],
$$


and we have $\operatorname{det}[P(u)]=1 / u^{4}+4$ which has degree $4=N(P)$. Thus $P$, regular. Now by applying Sumudu transform to the above system we have

$$
P(u) S\left(\begin{array}{l}
x \\
y
\end{array}\right)(u)=\left[\begin{array}{c}
-\frac{u}{u^{2}+1} \\
1+2 u^{2} \\
-\frac{u^{2}+1}{2}
\end{array}\right]+\Psi_{P}(u) \phi(y, N(P))
$$

where $\Psi_{P}(u) \phi(y, N(P))$ is given by

$$
\Psi_{P}(u) \phi(y, N(P))=\left[\begin{array}{cccc}
\frac{1}{u^{2}} & \frac{1}{u} & \frac{1}{u} & 0 \\
-\frac{1}{u} & 0 & \frac{1}{u^{2}} & \frac{1}{u}
\end{array}\right]\left[\begin{array}{l}
1 \\
2 \\
0 \\
1
\end{array}\right]=\left[\begin{array}{c}
\frac{1+2 u}{u^{2}} \\
-\frac{1}{u}
\end{array}\right]
$$

On using (1.25) we obtain

$$
P(u)^{-1}=\left[\begin{array}{cc}
\frac{u^{2}\left(1-2 u^{2}\right)}{1+4 u^{4}} & \frac{-2 u^{3}}{1+4 u^{4}} \\
\frac{2 u^{3}}{1+4 u^{4}} & \frac{u^{2}\left(1-2 u^{2}\right)}{1+4 u^{4}}
\end{array}\right]
$$

then equation (1.45) becomes

$$
S\left(\begin{array}{l}
x \\
y
\end{array}\right)(u)=P^{-1}(u)\left[\begin{array}{c}
-\frac{u}{u^{2}+1} \\
1+2 u^{2} \\
-\frac{u^{2}+1}{2}
\end{array}\right]+P^{-1}(u)\left[\Psi_{P}(u) \phi(y, N(P))\right]
$$

finally, by taking inverse Sumudu transform equation (1.48) we obtain the solution of the system as follows

$$
\begin{aligned}
& x(t)=\sin (t)+e^{t} \cos (t), \\
& y(t)=-\cos (t)+e^{t} \sin (t)+1
\end{aligned}
$$

Thus based on the above discussions we note that the Sumudu transform can be applied for system of differential equations thus can be used in many engineering problems.

\section{Acknowledgments}

The authors gratefully acknowledge that this research was partially supported by the University Putra Malaysia under the Research University Grant Scheme 05-01-09-0720RU and Fundamental Research Grant Scheme 01-11-09-723FR. The authors also thank the referee(s) for very constructive comments and suggestions. 


\section{References}

[1] G. K. Watugala, "The Sumudu transform for functions of two variables," Mathematical Engineering in Industry, vol. 8, no. 4, pp. 293-302, 2002.

[2] G. K. Watugala, "Sumudu transform: a new integral transform to solve differential equations and control engineering problems," International Journal of Mathematical Education in Science and Technology, vol. 24, no. 1, pp. 35-43, 1993.

[3] S. Weerakoon, "Application of Sumudu transform to partial differential equations," International Journal of Mathematical Education in Science and Technology, vol. 25, no. 2, pp. 277-283, 1994.

[4] M. A. Asiru, "Sumudu transform and the solution of integral equations of convolution type," International Journal of Mathematical Education in Science and Technology, vol. 32, no. 6, pp. 906-910, 2001.

[5] A. K1lıçman and H. E. Gadain, "An application of double Laplace transform and double Sumudu transform," Lobachevskii Journal of Mathematics, vol. 30, no. 3, pp. 214-223, 2009.

[6] F. B. M. Belgacem, "Boundary value problem with indefinite weight and applications," Problems of Nonlinear Analysis in Engineering Systems, vol. 10, no. 2, pp. 51-58, 1999.

[7] A. Kadem, "Solving the one-dimensional neutron transport equation using Chebyshev polynomials and the Sumudu transform," Analele Universitatii din Oradea. Fascicola Matematica, vol. 12, pp. 153-171, 2005.

[8] H. Eltayeb and A. Kılıçman, "On double Sumudu transform and double Laplace transform," Malaysian Journal of Mathematical Sciences, vol. 4, no. 1, pp. 17-30, 2010

[9] A. K1lıçman and H. Eltayeb, "A note on integral transforms and partial differential equations," Applied Mathematical Sciences, vol. 4, no. 3, pp. 109-118, 2010.

[10] H. Eltayeb and A. Kılıçman, "On some applications of a new integral transform," International Journal of Mathematical Analysis, vol. 4, no. 3, pp. 123-132, 2010. 


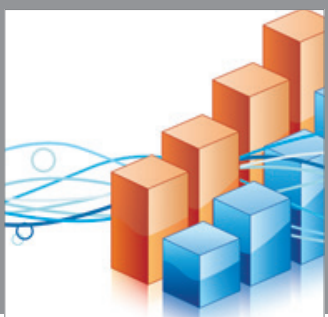

Advances in

Operations Research

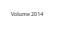

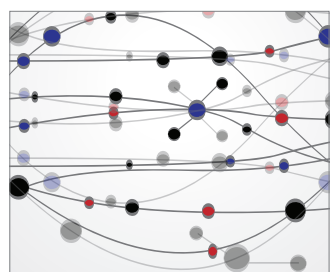

\section{The Scientific} World Journal
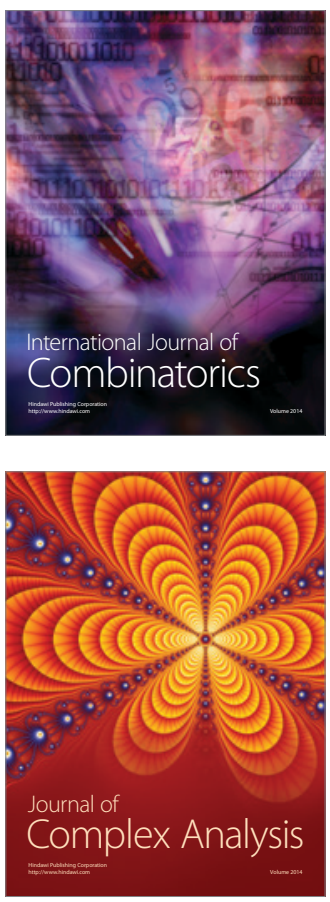

International Journal of

Mathematics and

Mathematical

Sciences
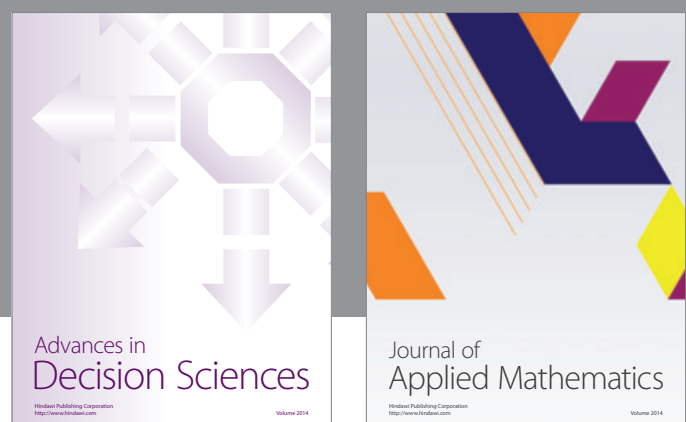

Journal of

Applied Mathematics
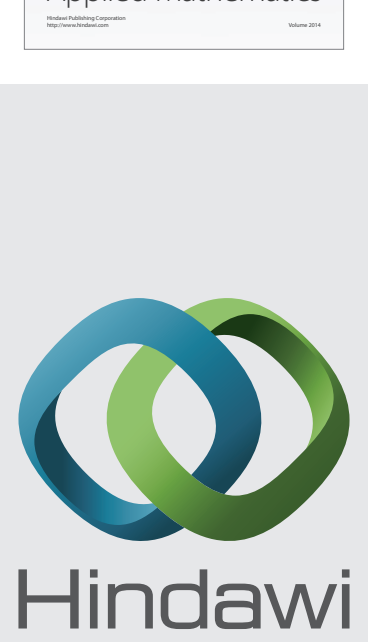

Submit your manuscripts at http://www.hindawi.com
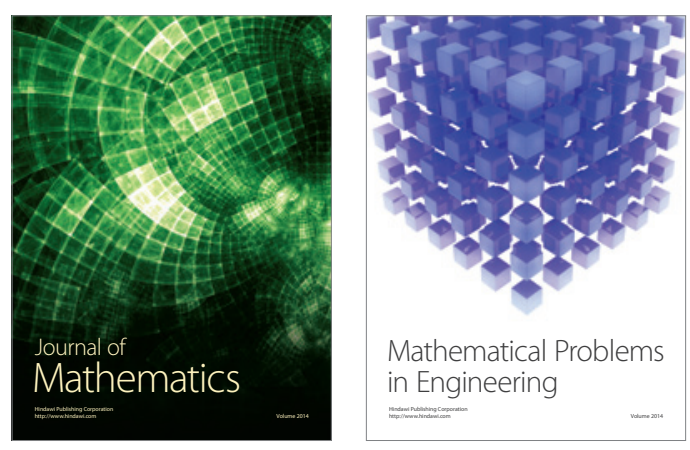

Mathematical Problems in Engineering
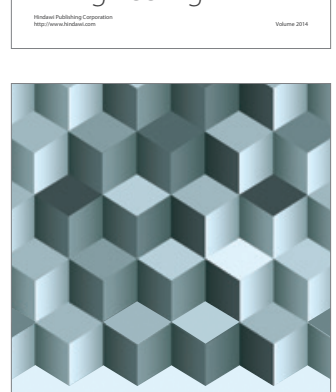

Journal of

Function Spaces
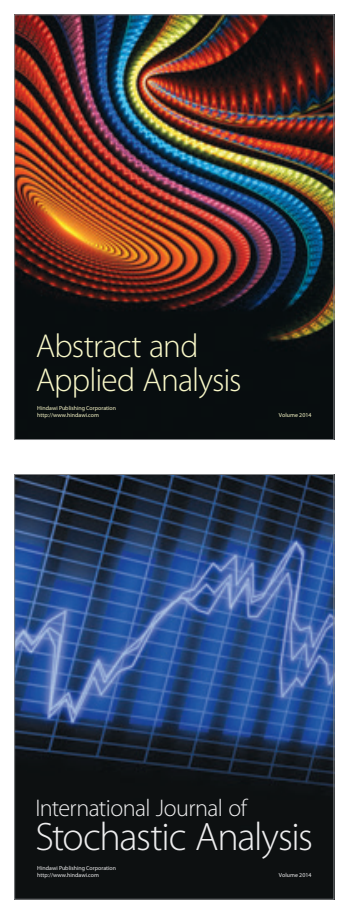

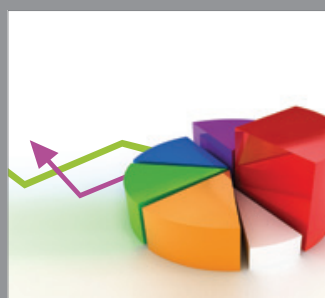

ournal of

Probability and Statistics

Promensencen
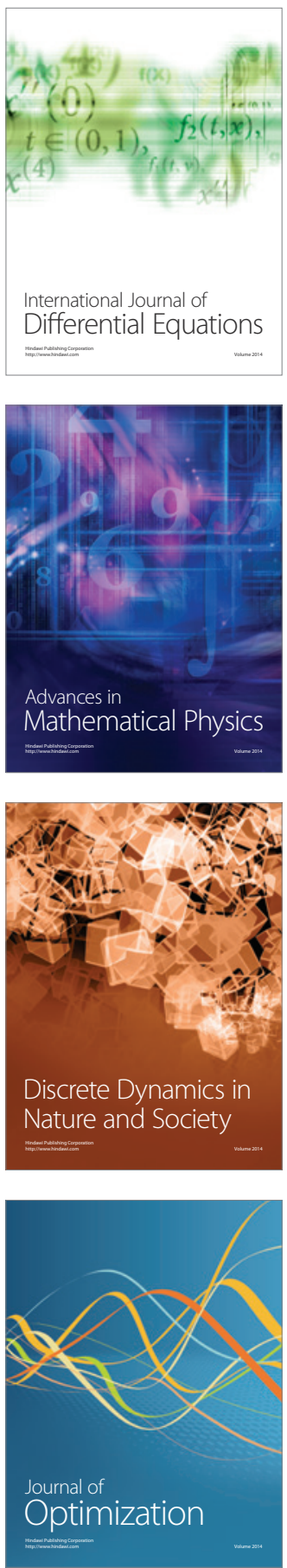